\title{
A Study on the Word Formation of Noun-Classifier Compound
}

\author{
Words* \\ CUI Yan-ji, ZHAO Lei, AN Feng-cun \\ Collage of Foreign languages, Yanbian University, Yanji City, Jilin Province, 133002, China
}

\begin{abstract}
Chinese noun-classifier compound words show different properties in lexical meaning and syntactic collocation. The complexity of these compounds lies in the grammaticalization of classifiers' system. Some nouns used as classifiers in the position of classifier are not grammaticalized into real classifiers which are temporary classifiers. So some of them are real noun-classifier compounds and some belong to noun-noun compounds. This paper attempts to analyze the structural relation between noun morpheme and classifier morpheme within the word. It also explains that the noun and the classifier compound in the position of light noun in the $\mathrm{nP}$ structure.
\end{abstract}

Keywords: word formation, noun-classifier compound words, light noun

\section{Introduction}

In Chinese, there exist a group of compounds with noun-classifier compositional elements, such as cheliang (cars), mapi (horses), qiangzhi (guns), chuanzhi (ships), fangjian (rooms), zhizhang (paper), bupi (cloth), shuben (books), wujian (objects), renkou (population), huaduo (flowers), dengzhan (lamps), shipian (poems), shijian (accidents), shiju (poems), shujuan (books), tianmu (fields), yinliang (money), etc. There are two elements in the above words: the former noun morpheme and the later classifier morpheme. In some Chinese studies, if compound words have the noun-classifier sequence, then they can be grouped into the same type of compounds. So the words like shabao (sand-bag), renqun (person-group), even fanwan (rice-bowl) are all put into the same word sort. However, the differences among this kind of compounds can be easily found from the meanings of words. For example, the word mapi (horses) tends to express the meaning of ma (horse) morpheme; while the word shabao (sand-bag) tends to denote the meaning of the morpheme bao (bag). The simple comparison shows that two words with the seemingly same elements and structures are quite different in the denotation.

What are the criteria to sort out the type of these compounds? What is the relation between the meaning of the compound and its internal structure? How to explain the differences between the compounds such as mapi (horses) and shabao (sand-bag)? The paper will tend to solve the above problems from the property of classifiers and the structure of the two morphemes in Chinese noun-classifier compound words (hereafter referred to as

* Acknowledgments: This paper is supported by Jilin Social Science Fund of China (Grant No. 2018B34), The Ministry of Education of Humanities and Social Science project (Grant No. 16YJC740055).

CUI Yan-ji, Lecturer, College of Foreign Languages, Yanbian University. Research fields: syntax.

ZHAO Lei, Doctoral candidate, College of Foreign Languages, Yanbian University. Research fields: syntax.

AN Feng-cun, Associate Professor, College of Foreign Languages, Yanbian University. Research fields: linguistic theory and syntax. 
$\mathrm{N}-\mathrm{Cl}$ compounds).

\section{The Syntactic Relation between Morphemes in N-Cl Compounds}

Most previous studies on $\mathrm{N}-\mathrm{Cl}$ compounds in Chinese focus on the syntactic relation between the two morphemes in the word. It shows that scholars have been noticing the nature of the words and the uniqueness of this structural pattern. The following are the points of view on the structural relation of Chinese $\mathrm{N}-\mathrm{Cl}$ compounds.

\section{The Head-complement Structure}

The point is held by the scholars such Huang Borong \& Liao Xudong (2002), Ge Benyi (2001), etc. For example, for the words huaduo (flowers), mapi (horses), and fangjian (rooms), they believe that the former part which denotes an entity is the head element of the word and the latter element is the measurement unit of such entity.

Huang \& Liao (2002) hold this view and also try to prove and explain the structure of noun-classifier compounds by comparing them with the head-complement compound words like tigao (lift-high), tuifan (push-over), chanming (explain-clear), etc. And they further classify the head-complement structure into two groups on the basis of the category of the first morpheme in the head-complement compound words. One is the verb compound with an action as head and a resultant as complement, the other is the noun compound with an entity as head and a complement to quantify the entity.

\section{The Modifier-head Structure}

The view is that there is a special modifier-head relation between two morphemes in N-Cl compounds which is held by Zhao Yuanren (1979), Zhu Dexi (1982), etc.

Zhao (1979) believes that such group of words are attributive word, which belongs to N-M (N is a nominal morpheme, $\mathrm{M}$ is a measuring morpheme). According to him, such N-M is a structure in which the nominal morpheme modifies the classifier morpheme. The classifier is the head of the whole word structure.

Other scholars also mention that the relation between noun morpheme and classifier morpheme is the noun-noun relation in nature. They point out that the classifier can involve the formation of the compound words as an abstract noun, and that the nature of classifier in this condition is a noun.

\section{The Stem-affix Structure}

Ma Qingzhu (1987) and others hold the point that the classifier morpheme is an affix attached to the N stem in $\mathrm{N}-\mathrm{Cl}$ compounds.

There is a kind of word-formation which adds a classifier at the end of the noun. The reason why people consider it as a method of word-formation is that the classifier does not function as the quantity because of grammaticalization. For example, the word maqun (a herd of horses), huashu (bouquet) and youjian (letters) are all stem-affix structures.

Ma (1987) distinguishes affix and pseudo affix. Affixes added to the end of the noun are real affixes. He further explains that real affixes are transformed from the classifiers and grammaticalization.

\section{The Coordination Structure}

Li Zongjiang (2004) points out that the noun-classifier compound structure is a coordinate structure; he also gives three arguments to support his point. 
Firstly, the classifier which always appears with certain noun gradually acquires the meaning of the noun and finally can refer to the noun independently.

Secondly, the classifier always matches with a noun. It manifests a kind of selectivity. Some classifiers possessing a range of selectivity, such as ge (a), mei (a) in Chinese, cannot easily acquire the meaning of certain noun.

Thirdly, most of these words, such as fangwu (houses), liangshi (food) and qiangbi (walls), are similar to the compound words which are generated by synonymous morphemes.

\section{Comments on Previous Studies}

Different researchers have different opinions on the structure of the $\mathrm{N}-\mathrm{Cl}$ compounds.

Different kinds of $\mathrm{N}-\mathrm{Cl}$ compounds are formatted from different processes. It seems that this kind of compounds have the same surface composition with a noun morpheme and a classifier morpheme. The surface of the compound shows that the noun-classifier linear series is same; however, the words can not be sorted out just according to the compositional elements and the serial order of noun morpheme and classifier morpheme. The classifier morpheme is the key to judge the type of a compound with a noun morpheme and a classifier morpheme.

Only when the reasonable and scientific criterion is set to sort out the compounds with noun-classifier composition, can the internal structure and the meaning of the words be analyzed.

\section{The Classification of N-Cl Compounds}

These N-Cl compounds don't follow the same rule of the word formation. The word such as mapi(horses) denotes the generic reference of the correspondent noun morpheme in the compounds. When the noun morpheme and the classifier morpheme appear in quantity phrase like yipima(a horse), they have a fixed relation of collocation.

The evolution of Chinese classifiers obeys the following stages: lexical words $\rightarrow$ functional words $\rightarrow$ clitics $\rightarrow$ affixes, which is the main reason to make the Chinese $\mathrm{N}-\mathrm{Cl}$ compounds more complex. And there are lots of temporary classifiers loaned from nouns which also expand the members of the $\mathrm{N}-\mathrm{Cl}$ compounds. Therefore, the classifier is the key element to sort out the $\mathrm{N}-\mathrm{Cl}$ compounds and classify the compounds.

The structural relation between the morphemes in these compounds should be paid attention to. First, the classifier-noun collocation in the phrase is the basis to form $\mathrm{N}-\mathrm{Cl}$ compounds, such as mapi (horses). The phrase yipima (one horse) in Chinese can show the collocation between pi and ma. Second, the degree of grammaticalization of classifiers can determine the core element in $\mathrm{N}-\mathrm{Cl}$ compounds, such as in mapi, ma functions as the core element; however, in the word chenggan (scale-beam), gan is the core element which is not a classifier but a noun.

According to the above criteria, there are three main types of the N-Cl compounds.

\section{The Noun Morpheme as the Core Semantic Element}

The semantic core in this kind of $\mathrm{N}-\mathrm{Cl}$ compound refers to the noun morpheme such as bupi (cloth), cheliang (cars), chuanzhi (ships), dengzhan (lamps), which denotes the generic reference and can't be matched with numeral-classifier determiners (see Example (1)). 


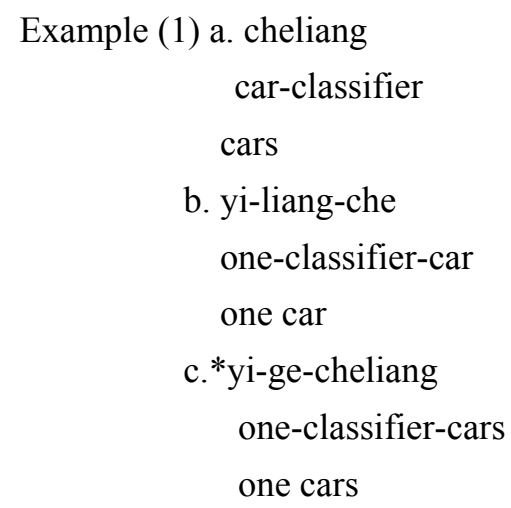

The compound with noun morpheme as the core element has the collective denotation, which is contradicted to the concrete quantity expressed by numerals.

\section{The Classifier Morphemes as the Core Semantic Element}

In this type of the compounds, the classifier morpheme is the core semantic element and the whole compound tends to denote the meaning of the classifier morpheme. It shows that in this condition, the classifier in the noun-classifier structure tends to be a noun, such as chenggan (scale-beam), quanyan (spring-eye). The above words tend to express the meaning of the classifier element which refers to the meaning of the classifier as a noun. And we can also find in this type of the noun-classifier words, the classifier element shows much more nominal properties beyond classifier property. And this group of the words can be quantified by numerals, see Example (2):

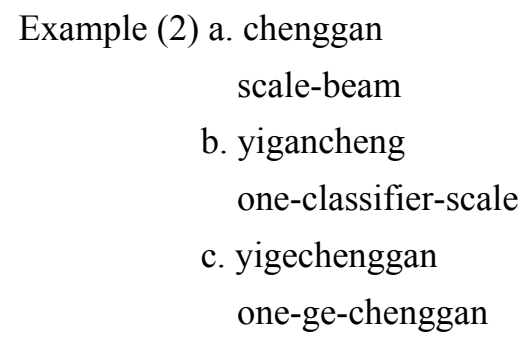

So the classifier element in compounds refers to its original meaning as a noun, but not functions as the grammaticalized classifier.

\section{No Morpheme as a Core Semantic Element}

Some words with noun-classifier structure have no obvious core element, such as bankuai (board-chunk), guapian (melon-piece), qianggan (gun-barrel), shipian (poem-chapter), shujuan (book-volum). The meanings of above words have shifted from the original meaning and developed new meanings. For example, bankuai (board-chunk) has no business with ban (board) or kuai (chunk), guapian refers to a kind of tea from which nothing can be found about gua or pian, qianggan is the metonymy of weapon, shipian and shujuan both denote the articles.

So from the classification of noun-classifier compounds, they can be put into different word groups and be analyzed according to their structures and meanings respectively. A true sense of noun-classifier compound only covers the type in 4.1 , and the words in 4.2 belong to N-N compound with modifier-head relation in the words. 


\section{The Syntactic Analysis of N-Cl Compounds}

\section{The Nature of Classifiers}

About the word class of Chinese classifier, traditional Chinese studies regard classifiers as measure words which combine with numerals or demonstratives to modify nouns and express the meaning as units. In the field of Generative Grammar, researchers put classifier in the functional category which has its own projection CIP (classifier Phrase), such as the studies from Li (1999), Cheng \& Sybesma (1999), Cheng \& Sybesma (2005), Jiang (2012), etc.

An (2009), An \& Cheng $(2011,2014)$ believe that the method of CIP above has strong pertinence. On one hand, it aims at Chinese classifier, and only analyzes the syntactic structure of Chinese classifier phrases; on the other hand, it doesn't explain the morph-syntactic property of Chinese classifiers and it only gives a structural interpretation.

According to the descriptions of the functional category by Napoli (1993), classifiers belong to the functional category for the following reasons.

First, classifiers belong to the closed class. Some scholars also investigate the Chinese classifiers and sort out the individual classifiers which are so called typical classifiers. They are different from measure words. According to the investigation, the classifiers are not beyond 50 in modern Chinese, such as "ge, tiao, mei, zhi, pi, ben, kou, tou, zhi, tai, jian, pian, bu, gen, etc." And ge is becoming an almighty classifier, so the amount of classifier is shortened. Therefore the grammatical function of classifier is much more important than its semantic function. Second, when a classifier is chosen to match a noun, the collocation between the classifier and noun should be taken into consideration; while, in fact, classifiers don't have the property of semantic selection. Third, classifiers have a function of categorical selection. In the structure, classifier selects and only selects a noun, and the words in other categories can be selected by the classifier as its complement. Last, classifiers are not free morpheme, and they are cohesive.

All the features above are typical features of functional category. It is obvious that classifiers belong to the functional category. There are still some questions. What kind of category is it? What is the syntactic position of classifier? What is the property? And what is the function?

In order to know the properties of classifiers, all the phenomena of classifiers should be taken into consideration. The changes of classifiers' functions in sentence should be clear. The difference between classifiers and measure words should be identified from its original positions. Besides, the reason why classifiers have the similar syntactic forms and differ from them should be explained.

As for the properties of Chinese classifiers, different scholars give different understandings and points of view. Here, this paper adopts the idea by An \& Cheng $(2011,2014)$ from Generative Grammar. The difference between Chinese classifiers, such as ben, zhi, tiao and measure words, such as bei, bao, xiang should be recognized. Measure words are the units to quantify the uncountable nouns and describe the countable nouns with collective concept.

An \& Cheng $(2011,2014)$ point out that Chinese classifiers originate from nouns under special environments. Only countable nouns can be the source for classifiers to deprive from, from which the relations between classifiers and countable nouns can be found, and further the property and function of classifiers can 
be understood. According to them, Chinese classifiers are light nouns which mark the countability of the corresponding noun. The classifiers can also substitute the corresponding noun just because they derive from countable nouns.

Classifiers originate from nouns, so they must have certain relationship in the nominal structure, which can be called DP. From the evolutional process of Chinese classifiers, we believe that there exists a null morphological element which is functional and finally has the morphological form. That is the light noun.

In the view of this, the specific morph-syntactic property of Chinese classifier is " $n$ ", which occupies the head position in the structure. The classifier owns whole properties and features of functional category. It is good to the research of how classifier noun construction embodies the function and meaning of the determiner.

\section{The nP Structure for Chinese Classifiers}

An \& Cheng (2011) [2] set up an nP structure, which possesses the following functions: 1) explain the emergence of classifiers; 2) differ from measure words; 3) explain the changes of classifier's function in the structures; 4) clear the syntactic distribution; 5) deal with the changes of quantity structure uniformly. The hypothesis also unifies the nominal structure of languages, and is not limited to certain languages. It will bring benefits by dealing with classifiers from generative aspect.

Example (3) nP Structure for Chinese classifier

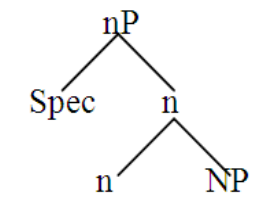

In the structure above, the classifier generates at the position of $\mathrm{n}$, which is the head of $\mathrm{nP}$. Numeral is at the position of [Spec $n]$. According to the relationship of category selection between classifiers and nouns, NP appears after the classifier all the time. This is why we see "n", which is at the position of the classifier, choose $\mathrm{NP}$ as its complement. This kind of structure accords with the relation between classifiers and nouns. Besides, numeral belongs to the open class, which means that it does not possess the features of functional category. Therefore, it must appear at the position [Spec n]. From the logic perspective, only the [+count] feature of noun has been checked by the classifier, can numerals be combined with the position [Spec, n], and the meaning of number can be expressed from the structure. Otherwise, numerals cannot appear at this position. In order to illustrate it clearly, we take the word in 4 as Example (4):

$\begin{array}{rlll}\text { Example (4) yi } & \text { ben shu } \\ \text { one } & \mathrm{Cl} & \text { book } \\ \text { one } & \text { book } & \end{array}$

In this phrase, as shown above, the classifier is the head of the phrase, after which there exists the NP (book). Before the numeral yi (one) combines with [Spec $n]$, the classifier needs to check the [ + count] feature of the NP (book). When it confirms that the NP is countable, the numeral can get its position to express the meaning of number of this phrase.

\section{The nP Structure for Noun-classifier Compounds}

This paper proposes that the Chinese $\mathrm{N}-\mathrm{Cl}$ compounds have a syntactic environment to help the words to derive from, and the reason why noun and classifier can compound is that the change of linear order of 
numeral-classifier and noun. The traditional order is noun-numeral-classifier order, and then the order changes into numeral-classifier-noun order. However both of them have the same syntactic structure. When the linear order changes, especially when the numeral is one, the numeral one can be omitted in utterance, which builds the environment for noun-classifier sequence and classifier-noun sequence. The numeral-classifier-noun becomes the standard structure of Chinese quantity structure, even the numeral one is null, the classifier-noun sequence can't form a word. However, the noun-classifier sequence exists in Chinese, and it develops the new function which can distinguish it from the classifier-noun sequence. There are still questions that need to be explained: what is the difference between the noun-classifier sequence with a null numeral and the noun-classifier compound? Where do they form in the syntactic structure?

Traditional noun-classifier sequence like mapi (horse-classifier) is a syntactic phrase structure with the drop of the numeral yi (one), which means "horse is one". The following structure is the syntactic structure with traditional shuben (book-classifier) as a case.

Example (5) Tradition sequence of noun-classifier

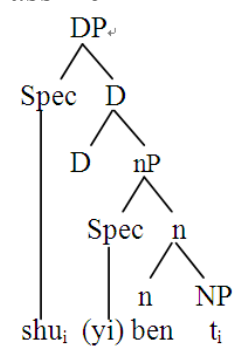

In the traditional sequence of noun-classifier, there is a syntactic relation between noun and classifier without the word yi (one). So in this condition, shu (book) moves to the position of [Spec D] which leaves the default position of $y i$ (one) in the position of [Spec n]. However, after the noun-numeral-classifier sequence changes into numeral-classifier-noun order, the noun-classifier must have a new function which is the word formation. They still have the syntactic base as follow:

Example (6) Compound of noun-classifier

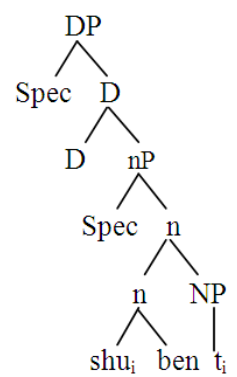

In this condition, shu (book) moves to the position of $\mathrm{n}$ and compounds with $\mathrm{n}$ to form a new syntactic unit, that is shuben, and then shuben as a whole moves to D to acquire the generic reference with the collective denotation.

Therefore, the traditional noun-classifier sequence and the noun-classifier compound have different structures and generate in the different syntactic position.

\section{Conclusion}

Chinese $\mathrm{N}-\mathrm{Cl}$ compounds in fact only cover a limited group of words, such as shuben (books), which have 
syntactic bases. After Chinese quantity structure noun-numeral-classifier changes into numeral-classifier-noun, the noun-classifier sequence must change the structural relation from the phrasal relation into the word-internal relation.

\section{References}

An, F. C. (2009). On interior adjustment of language structure from the grammaticalization of classifiers (in Chinese). Chinese Language Learning, (4), 56-60.

An, F. C., \& Cheng, G. (2011). A study on morphosyntactic properties of chinese classifiers (in Chinese). Chinese Language Learning, (2), 44-47.

An, F. C., \& Cheng, G. (2014). A study on syntactic function of Chinese classfiers in generative perspective (in Chinese). Journal of PLA University of Foreign Lnaguages, (2), 51-58.

Cheng, L. L.-S., \& Sybesma, R. (1999). Bare and not-so-bare nouns and the structure of NP. Linguistic Inquiry. (4), 509-542.

Cheng, L. L.-S., \& Sybesma, R. (2005). Classifers in four varieties of Chinese. In G. Cinque and R. Kayne (Eds.), The Oxford handbook of comparative syntax. Oxford: Oxford University Press.

Cheng, L. L.-S., Sybesma, R., Tang, Y.-W., \& Tang, Y.-G. (1998). Classifiers and massifiers (in Chinese). The Tsing Hua Journal of Chinese Studies, (3), 385-412.

Croft, W. (1994). Semantic universals in classifier system. Word, (2), 145-171.

Huang, B. R., \& Liao, X. D. (2002) Modern Chinese language (in Chinese). Beijing: Higher Education Press.

Iljic, R. (1994). Quantification in Mandarin Chinese: Two markers of plurality. Linguistics, (1), 91-116.

Jiang, L. (2012). Nominal argument and language variation (Dissertation, Harvard University).

Li, Y.-H. A. (1999). Plurality in a classifier language. Journal of East Asian Linguistics, (1), 75-99.

Li, Z. J. (2004). Counterprocess in grammaticalization and the lexicalization of Chinese classifiers (in Chinese). Research in Ancient Chinese Language, (4), 62-67.

Longobardi, G. (1994). Reference and proper names: A theory of N-movement in syntax and Logical Form. Linguistic Inquiry, (4), 609-665.

Ma, Q. Z. (1987). The essence, grammatical function and application of abbreviations (in Chinese). Language Teaching and Linguistic Studies, (3), 20-27.

Pan, H. H., \& An, F. C. (2012). A comparative study on the core syntactic hierarchies of English and Chinese noun phrases (in Chinese). Foreign Language Teaching and Research, (5), 658-670.

Wang, J., \& Gu, J. S. (2006). A study on the special usages of classifiers in Lianshui dialect of Chinese (in Chinese). Studies of the Chinese Language, (3), 237-241.

Zhao, Y. R. (1979). The grammar of Chinese spoken language (in Chinese). Beijing: The Commercial Press.

Zhu, D. X. (1982). Lectures on Chinese grammar (in Chinese). Beijing: The Commercial Press. 